\title{
Boosting Light Emission from Single Hydrogen Phthalocyanine Molecules by Charging
}

\author{
Vibhuti Rai,* Lukas Gerhard, Qing Sun, Christof Holzer, Taavi Repän, Marjan Krstić, Liang Yang, \\ Martin Wegener, Carsten Rockstuhl, and Wulf Wulfhekel
}

\begin{abstract}
Interest in electroluminescence of single molecules is stimulated by the prospect of possible applications in novel light emitting devices. Recent studies provide valuable insights into the mechanisms leading to single molecule electroluminescence. Concrete information on how to boost the intensity of the emitted light, however, is rare. By combining scanning tunnelling microscopy (STM) and quantum chemical calculations, we show that the light emission efficiencies of an individual hydrogen phthalocyanine molecule can be increased by a factor of $\approx 19$ upon charging. This boost in intensity can be explained by the development of a vertical dipole moment normal to the substrate

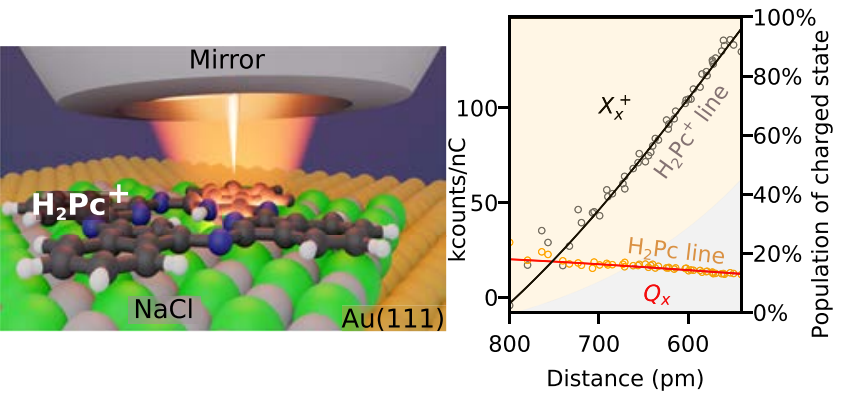
facilitating out coupling of the local excitation to the far field. As this effect is not related to the specific nature of hydrogen phthalocyanine, it opens up a general way to increase light emission from molecular junctions.
\end{abstract}

KEYWORDS: Organic light emitting diodes, scanning tunnelling microscopy, scanning tunnelling microscopy induced luminescence, direct laser writing, quantum chemical calculations

$\mathrm{T}$ he possibility to convert an electric current into light is highly attractive from a scientific point of view as well as with regard to its application as a light source. Driven by the development of organic light emitting diodes (OLEDs), intensive efforts have been made to understand the fundamental mechanisms involved in the light emission processes from organic layers by scaling down the system under study to a single molecule adsorbed on a surface. Scanning tunnelling microscopy induced luminescence (STML) provides a unique experimental route to study electrically induced light emission and underlying mechanisms at the single molecule level. ${ }^{1-7}$ Recently, it has allowed the discovery of many intriguing phenomena such as vibrationally resolved fluorescence, hot electroluminescence, Fano reso nance effects, plasmon-exciton coupling, dipole-dipole coupling, up conversion electroluminescence, and molecular electrofluorochromism from single molecules. ${ }^{2-4,8-11}$ How ever, a general concept of how to increase the overall efficiency of light emission is lacking, although it is a key factor when it comes to possible applications. Here, we use STML to study the electroluminescence intensity of dihydrogen phthalocya nine $\left(\mathrm{H}_{2} \mathrm{Pc}\right)$ adsorbed on $\mathrm{NaCl} / \mathrm{Au}(111)$. Supported by quantum chemical calculations, we assign a newly found bright emission line to the charged molecule. Using a population model of the molecule in the charged and uncharged state, we show that the photon emission of the charged molecule is 19 times more efficient. By comparison with quantum chemical and full wave optical calculations, we trace the boost back to the vertical dipole moment that evolves with the structural deformation upon charging. Full wave optical calculations reveal that this vertically oriented dipole moment relative to the STM junction drastically improves out coupling of the radiation extracted from the molecule. These findings explain the experimentally observed high efficiency luminescence.

In an OLED device, the optically active molecules are placed in an electric junction in order to drive electroluminescence. It is paramount to utilize injection layers for the charge transport to avoid nonradiative recombination that quenches the electroluminescence. Here, we take the common approach to electrically decouple the $\mathrm{H}_{2} \mathrm{Pc}$ molecules from the $\mathrm{Au}(111)$ substrate by virtue of a thin insulating layer. ${ }^{2,11,12}$ Besides reducing nonradiative recombination, the layer also allows for long lifetimes of charged states that impact the transition lines in electroluminescence as has been observed for $\mathrm{ZnPc}$ molecules on thin $\mathrm{NaCl}$ layers. ${ }^{4}$

Figures $1 \mathrm{a}$ and $1 \mathrm{~b}$ show the schematic of the experimental STML setup (not to scale, for details see SI Note 1) and a 

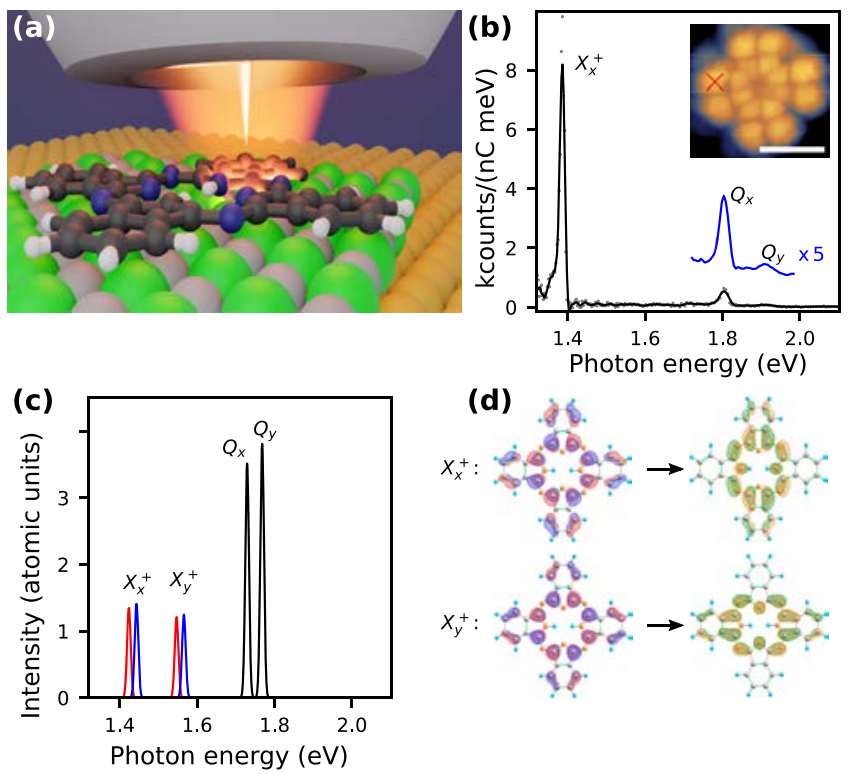

Figure 1. Electroluminescence and simulation of emission lines of $\mathrm{H}_{2}$ Pc. (a), Schematic of the experimental setup including a 3D printed parabolic micromirror for efficient light collection (tip and molecule are not to scale).$^{13}$ The single $\mathrm{H}_{2} \mathrm{Pc}$ molecules are decoupled from the $\mathrm{Au}(111)$ surface by three layers of $\mathrm{NaCl}$. They adsorb planar on the surface. (b) In black, the spectrum of the emitted photons recorded with the tip positioned at the edge of a $\mathrm{H}_{2} \mathrm{Pc}$ molecule (red cross in the inset topography) at negative sample bias $(U=-2.7 \mathrm{~V}, I=10 \mathrm{pA}$, $t=20 \mathrm{~s}$ ). To highlight the appearance of the high energy $Q_{x}$ and $Q_{y}$ lines, the spectrum is replotted at five times scale (blue line). Tunnelling parameters for the inset topography are $U=-2.2 \mathrm{~V}$ and $I$ $=2 \mathrm{pA}$, and the length of the scale bar is $1 \mathrm{~nm}$. (c) Calculated photoabsorption spectra of the uncharged $\left(Q_{x}\right.$ and $\left.Q_{y}\right)$ and the charged $\left(X_{x}^{+}\right.$and $\left.X_{y}^{+}\right)$molecule without (red line) and with (blue line) a negative point charge placed at the tip position. (d) Orbitals involved in the optical transitions of the charged system.

typical photon spectrum acquired at negative sample bias. In this report the typical quantum efficiency of the system is in the order of $\sim 10^{-5}$ photons/electron. ${ }^{14}$ Photon spectra presented in this report are corrected by detector efficiency, except for the losses in the parabolic mirror which are unknown (see Figure S1). The spectrum shows three peaks $\left(X_{x}^{+}, Q_{x}\right.$, and $\left.Q_{y}\right)$, of which the latter two can be assigned to the well known transition of the uncharged molecule from the lowest lying singlet states of $\mathrm{H}_{2} \mathrm{Pc}$ to the ground state. ${ }^{3,10,15-19}$ In contrast, the very bright and sharp $(\sim 11 \mathrm{meV}) X_{x}^{+}$peak has not been reported before. In a recent STML study on $\mathrm{ZnPc}$ on $\mathrm{NaCl} / \mathrm{Au}(111)$, an additional peak at similar energy could be assigned to the positively charged molecule. ${ }^{4}$

Motivated by these findings and with the purpose to understand the observed spectral lines, we performed quantum chemical simulations of the uncharged $\mathrm{H}_{2} \mathrm{Pc}$ and charged $\mathrm{H}_{2} \mathrm{Pc}^{+}$molecule (see SI Note 2). ${ }^{20-22}$ The gas phase spectra of molecular $\mathrm{H}_{2} \mathrm{Pc}$ (black line) and $\mathrm{H}_{2} \mathrm{Pc}^{+}$(red line) in Figure 1c reveal that the $X_{x}^{+}$excitation indeed stems from the charged molecule. Furthermore, the peak is split into two components, similar to the $Q_{x}$ and $Q_{y}$ lines of the uncharged molecule. Figure 1d shows the natural transition orbitals (NTOs) of the two calculated $X^{+}$excitations of the charged system. The NTOs reveal the character of the observed $X^{+}$transitions, assigning them to a transition from the highest lying occupied state to the lowest lying unoccupied state of the charged $\mathrm{H}_{2} \mathrm{Pc}^{+}$ molecule. Due to Coulomb interaction, the energetic position of the lines slightly shifts by the presence of nearby charge (blue line), used to reflect the presence of the tip next to the molecule. The calculation also reproduces the two exper imentally observed transition energies of the uncharged molecule. In the experiment, the higher energy line $Q_{y}$ is, however, less intense. This was attributed to the fast internal conversion of the excited $Q_{x}$ and $Q_{y}$ states. $^{23}$ Similarly, the higher energy line of the charged molecule $X_{y}^{+}$is not observed in the experiment on the level of the background intensity. Note that for a realistic description of the line intensities, the simulations would have to be refined to capture the geometry of the junction and to account for nonadiabtic excited state effects.

To further corroborate the nature of the new $X_{x}^{+}$spectral line, we measured photon spectra for both sample bias polarities (cf. Figure 2a). The $Q_{x}$ line appears for both

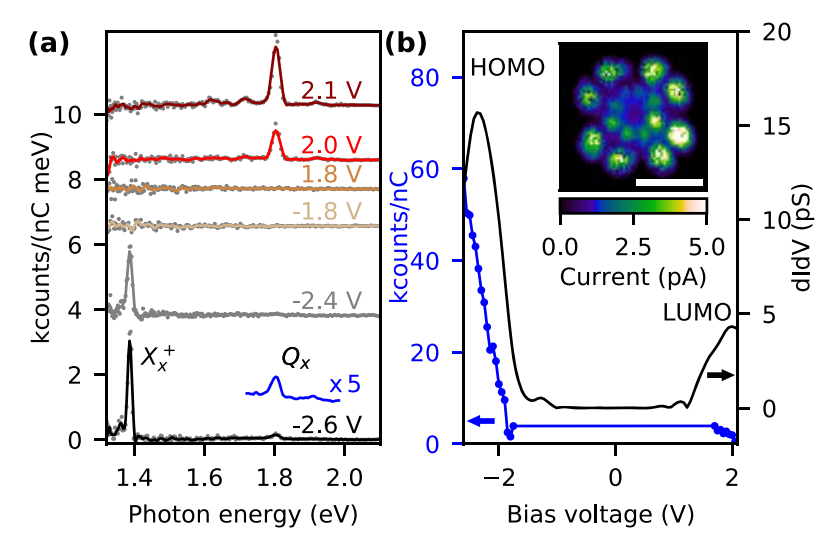

(c)

$Q_{x}(U>0)$

(d)

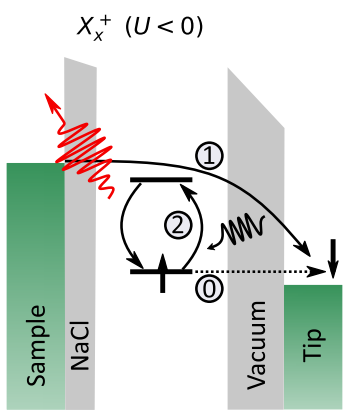

Figure 2. Energetics of the emission of the $X_{x}^{+}$line. (a) Photon spectra at different sample biases $(I=15 \mathrm{pA}, t=10 \mathrm{~s})$. (b) $\mathrm{d} I / \mathrm{d} U$ spectrum (black curve) recorded on a single $\mathrm{H}_{2} \mathrm{Pc}$ molecule and photon count integrated over the $X_{x}^{+}$peak as a function of sample bias (blue dots and line). The inset displays a current map of the $\mathrm{H}_{2} \mathrm{Pc}$ molecule recorded at constant height at $U=-2.3 \mathrm{~V}$. (c, d) Mechanisms for the emission of $Q_{x}$ and $X_{x}^{+}$lines. In both cases, a plasmon is excited by an inelastically tunnelling electron (process 1 ). The plasmon (black wavy arrow), in turn, excites the molecule (process 2 ) which decays under reemission of a photon (red wavy arrow). Emission of the $X_{x}^{+}$line (depicted in (d)) requires charging the molecule by elastic tunnelling of an electron from the HOMO to the tip (dotted arrow, process 0) prior to the excitation-emission cycle.

polarities at $|U| \geq 1.8 \mathrm{~V}$. This finding is in agreement with the established emission model in which a plasmon is inelastically excited by a tunnelling electron exciting the molecule followed by reemission (see Figure 2c). ${ }^{4}$ The dipole moment of the involved molecular excited states is oriented in plane, hampering emission to the far field and explaining the low 
(a)
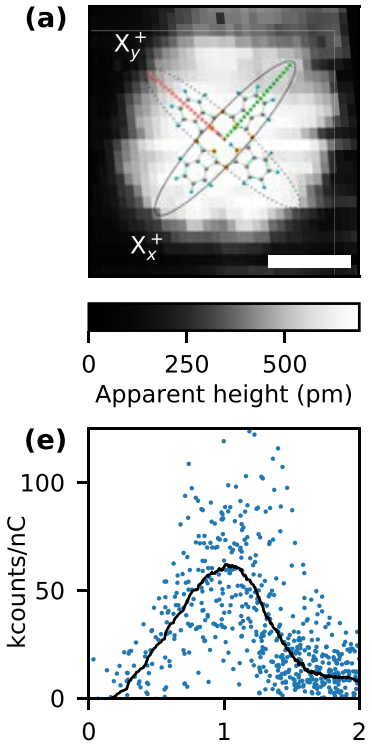

Distance from center $(\mathrm{nm})$ (b)

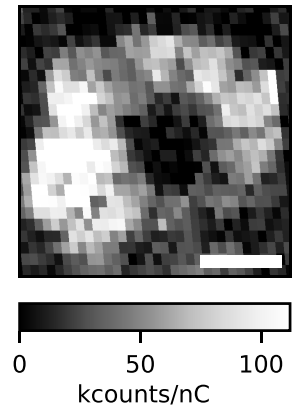

(f)

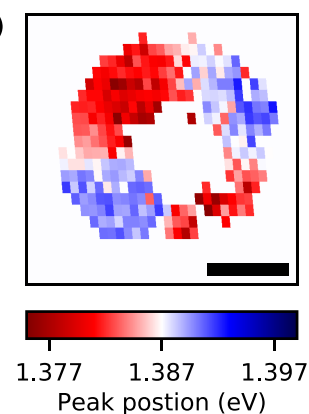

(c)
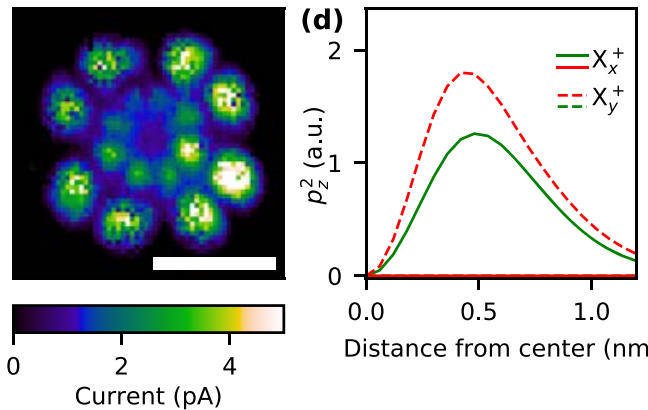

Distance from center $(\mathrm{nm})$

Current (pA)

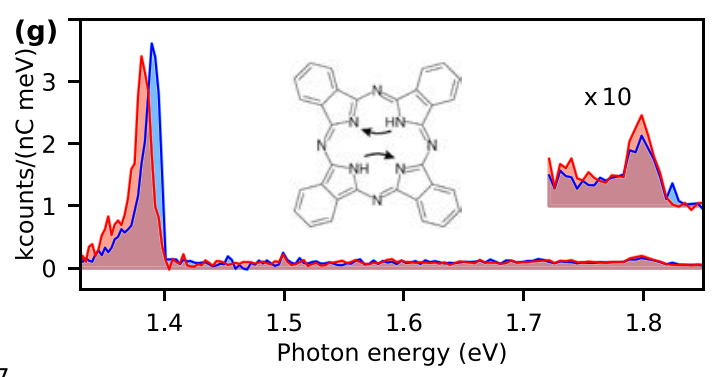

Figure 3. Spatial variation of the emission of the $X_{x}^{+}$line. (a) Topography of the $\mathrm{H}_{2} \mathrm{Pc}$ molecule during the recording of the photon map with molecular structure superimposed to scale. Solid (dashed) ovals indicate the orientation of the orbitals involved in light emission of $X_{x}^{+}\left(X_{y}^{+}\right)$lines. The length of the scale bar in (a) and (b) is $1 \mathrm{~nm}$. (b) Spatial distribution of integrated photon count in the energy range of the $X_{x}^{+}$line (1.373$1.401 \mathrm{eV}, U=-2.5 \mathrm{~V}, I=2 \mathrm{pA}, t=10 \mathrm{~s}$ ). (c) Current map of the $\mathrm{H}_{2} \mathrm{Pc}$ molecule recorded at constant height at $U=-2.3 \mathrm{~V}$ representing the HOMO of the molecule. (d) Green (red) lines depict the squared simulated out of plane component of dipole moment $p_{z}^{2}$ for a point charge (tip) located at the positions indicated by green (red) dots in (a). Solid (dashed) lines represent the $X_{x}^{+}\left(X_{y}^{+}\right)$emission lines. The red solid line lies above the green dashed line. (e) Integrated photon count of the $X_{x}^{+}$line for each pixel of the map in (b) (blue dots) and smoothed over 197 points (black line) as a function of distance from the center of the molecule. (f) The spatial distribution of the peak positions of the $X_{x}^{+}$spectral line shows four segments with two distinct peak positions, a low energy peak (red area) and a high energy peak (blue area). (g) Spectral lines averaged over the red and blue areas in (f).

intensity of the lines. Note that we did not observe a preference of emission of the $Q_{x}$ line for negative bias voltages as has been reported in the literature for $\mathrm{H}_{2} \mathrm{Pc}$ on $\mathrm{NaCl}$ on $\mathrm{Ag}(100) / \operatorname{Ag}(111))^{3,24} \mathrm{We}$, however, observed up conversion electroluminescence for $|U| \leq 1.8 \mathrm{~V}$ with low count rates (see Figure S6) as discussed recently for $\mathrm{H}_{2} \mathrm{Pc}$ on $\mathrm{NaCl}$ on $\operatorname{Ag}(100){ }^{3}$

The $X_{x}^{+}$line, in turn, is found only for negative sample bias in agreement with the charged state of the molecule. Emission sets in at $-1.8 \mathrm{~V}$ (Figure $2 \mathrm{~b}$, see also Figure S7). To understand the onset of the $X_{x}^{+}$line, we measured the electronic structure of the adsorbed molecule. Figure $2 b$ displays the integrated photon count of the $X_{x}^{+}$line as a function of $U$ together with the differential conductance (dI/ $\mathrm{d} U)$. The differential conductance shows two peaks at -2.3 and $1.7 \mathrm{~V}$ which we assign to the highest occupied molecular orbital (HOMO) and lowest unoccupied molecular orbital (LUMO), respectively. The inset of Figure $2 b$ shows a current map at constant height at $U=-2.3 \mathrm{~V}$ that nicely agrees with the calculated spatial distribution of the HOMO of a free $\mathrm{H}_{2} \mathrm{Pc}$ molecule (see Figure 1d). The energetic onset of the $X_{x}^{+}$line coincides with the HOMO peak, when electrons may tunnel elastically from the HOMO to the empty states of the tip charging the molecule. Thus, our observations agree with the established model for light emission of the charged species proposed by Doppagne et al. ${ }^{4}$ as depicted in Figure $2 \mathrm{~d}$, where charging the molecule is followed by inelastic excitation of a plasmon, absorption, and reemission into the far field. Thus, the intensity of the molecular lines is expected to be proportional to the plasmon intensity at the corresponding energy and the efficiency for emission into the far field. The plasmon resonance of the $\mathrm{Ag}$ tip used throughout this work is centered around $1.8 \mathrm{eV}$ (see Figure S1). Hence, the much higher intensity of the $X_{x}^{+}$line in comparison to the uncharged emission hints for a more efficient coupling of the charged molecule to the electric field, which will be discussed in more detail below. It is worth noticing that the $Q_{x}$ spectral line is present for negative voltages together with that of the charged state, indicating faster transitions between the differently charged states than our recording time $(\sim 1 \mathrm{~s})$. We would also like to mention that this simple plasmon energy transfer model might not be fully adequate to capture all physics involved in the light emission from the uncharged molecule., However, it allows us to describe the light emission from the charged species at negative sample bias and from the uncharged molecule at both bias polarities.

In agreement with the model, the spatial distribution of the intensity of the $X_{x}^{+}$line also reflects the HOMO. This is illustrated in Figures $3 a$ and $3 b$ which show the topography of a single $\mathrm{H}_{2} \mathrm{Pc}$ molecule and the light intensity of the emission at the $X_{x}^{+}$line on a grid of $36 \times 33$ pixels. ${ }^{11,25-27}$ Thus, the charging probability of the molecule as the initial step of the emission process is directly related to the energy and shape of the $\mathrm{HOMO}^{28}$ as is shown in Figure $3 \mathrm{c}$ and it enters the intensity of light emission. Note, however, that the inner lobes of the HOMO do not appear bright.

To understand the much brighter $X_{x}^{+}$line compared to the $Q_{x}$ line, we focus on the two models of the emission process. In both cases, the molecule absorbs a photon/plasmon and reemits it by coupling to the optical mode in the tunnelling 
junction. The optical transitions of the uncharged molecule involve molecular states with a dipole moment parallel to the surface. This hinders coupling to the electric field, which is mainly oriented normal to the surface. The situation for the charged molecule is, however, more complex.

As mentioned before, the spectrum of the charged species reacts on the presence of the tip, when mimicked by a point charge (see Figure 1c). When the molecule is charged, the molecular structure relaxes to the new ground state position of the charged system. This relaxation depends on, in addition to other effects, the static electric field in the biased tunnelling junction, i.e., it includes the mirror charge of the molecule in the $\mathrm{Au}(111)$ substrate and the field induced by the tip. We have carried out time dependent density functional theory calculations of this situation (see SI Note 2) to determine the dipole moment and its orientation as a function of the lateral tip positions as indicated in Figure 3a. The results for both charged lines are shown in Figure 3d. The calculations predict that the dipole moment $p$ is purely in plane ( $p_{z}$ vanishes) when the point charge is placed above the center of the molecule. Moving the point charge (tip) outward gives rise to a nonvanishing out of plane component $p_{z}$ of the dipole mo ment, which in turn results in improved propagation of light into the far field (see SI Note 3). This behavior can be compared to the radial dependence of the measured photon count presented in Figure 3e. The qualitative behavior agrees well, while the experimental curve is wider. This may be easily explained by the real tip being less sharp than an ideal point charge (see Figure S3). The $p_{z}^{2}$ is still low at the positions of the inner lobes of the HOMO, explaining the absence of these lobes in the experimental photon map. Note that when moving the tip perpendicular to the hydrogen bond direction (green lines in Figure 3d), the $X_{x}^{+}$line becomes bright and the $X_{y}^{+}$line vanishes. When going along the hydrogen bond direction (red lines in Figure $3 \mathrm{~d}$ ), the situation reverses. In the experiment, the $X_{y}^{+}$line is suppressed due to internal conversion. ${ }^{23}$ Moreover, the orbital from which the electron is taken cannot be independently chosen from the tip position when the plasmon is absorbed and re emitted. Thus, the experiment only reflects the solid $X_{x}^{+}$line of Figure 3d. Fast transitions of the hydrogen tautomers, as reported before, ${ }^{24}$ explain the light when moving the tip toward all eight lobes of the HOMO. In fact, we notice a small splitting of the $X_{x}^{+}$peak due to lifting of the symmetry of the molecule by the $\mathrm{NaCl}$ layer.

Figure $3 \mathrm{f}$ shows the spatial variation of the peak position of the $X_{x}^{+}$spectral line. This distribution map of peak positions shows four segments with two distinct peak positions, a low energy peak (red area) and a high energy peak (blue area). Figure $3 \mathrm{~g}$ shows the two spectral lines averaged over the red and blue areas in (Figure 3f) which are split by about $10 \mathrm{meV}$. In agreement with a recent study by Doppagne et al. ${ }^{24}$ on the uncharged $\mathrm{H}_{2} \mathrm{Pc}$, this behavior can be understood as the emission from two different tautomers of the charged $\mathrm{H}_{2} \mathrm{Pc}$ molecule with slightly different energies due to a different interaction with the substrate. For this, the local 4 fold symmetry of the $\mathrm{NaCl}$ layer in combination with the molecule needs to be broken. This may arise from the interaction with the $\mathrm{Au}(111)$ surface showing only 3 fold symmetry. The tautomerization of the charged neutral molecule was found to be triggered by inelastically tunnelling electrons and was on a much faster time scale than the recording time of the optical spectra. ${ }^{24,29}$ If this applies also to the charged molecule, we would observe light emission from the $X_{x}^{+}$spectral line from both of the tautomers and thus on all eight lobes of the molecule.

Our calculations show that the finite values of $p_{z}^{2}$ for the charged molecule are expected to enhance coupling to the far field. Combined with the requirement to extract an electron out of the HOMO, we can explain the sensitive emission behavior of the molecule upon placing the tip at a specific position relative to the molecule.

To quantify the difference in the quantum efficiencies of the $Q_{x}$ and $X_{x}^{+}$lines, we measured their intensities as a function of the tunnelling current $I$ at a fixed sample bias of $-2.5 \mathrm{~V}$ (Figure $4 \mathrm{a}$ and Figure S8). As we increase $I$, the intensities of
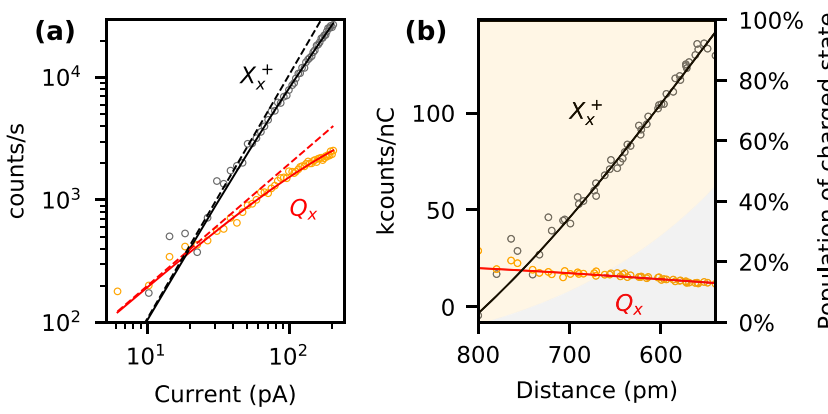

Figure 4. Current dependence of intensity and efficiencies of the charged and uncharged lines. (a) Intensities of the $X_{x}^{+}$(black dots) and $Q_{x}$ (orange dots) lines as a function of the tunnelling current at $U$ $=-2.5 \mathrm{~V}$. Black and red solid lines are fits according to the rate equations described in the text. Dotted lines are power law approximations. (b) Same data as in (a) normalized by tunnelling currents as a function of the distance between tip and molecule. The black (red) background depicts the population of the charged (uncharged) state.

both the $Q_{x}$ and $X_{x}^{+}$lines increase, but with different rates. In leading order (dashed lines in Figure $4 a$ ), the intensity of the $Q_{x}$ line scales linearly with current (one electron process, see Figure S9), while the $X_{x}^{+}$intensity has a quadratic dependency (two electron process) in agreement with the model. As charging is driven by electron tunnelling from the molecule to the tip across the vacuum barrier and uncharging by electron tunnelling from the sample across the $\mathrm{NaCl}$ layer, the change of the tunnelling current (vertical movement of the tip) mainly influences the charging probability and leaves the uncharging probability untouched. Increasing $I$ thus shifts the balance of the molecule to the charged state. As the molecule can either be charged or uncharged, the two emission lines compete. This can qualitatively explain the observed deviations from the simple power laws, i.e., with the increase of the charged population, the increase of the $Q_{x}$ line becomes weaker. We modeled the above described process using rate equations assuming that excitation into charged state is linear with current $(\alpha I)$, while the de excitation rate $(\gamma)$ back to the uncharged state is constant. In steady state, the populations for uncharged $\left(p_{\mathrm{u}}\right)$ and charged $\left(p_{\mathrm{c}}\right)$ states are then given as

$$
\begin{aligned}
& p_{\mathrm{u}}=\frac{\gamma}{\gamma+\alpha I} \\
& p_{\mathrm{c}}=\frac{\alpha j}{\gamma+\alpha I}
\end{aligned}
$$

The measured light intensities can then be expressed by $I_{\mathrm{u}}=$ $\eta_{\mathrm{u}} p_{\mathrm{u}} I$ and $I_{\mathrm{c}}=\eta_{\mathrm{c}} p_{\mathrm{c}} I$, where the "radiation efficiencies" $\eta_{\mathrm{u}}$ and $\eta_{\mathrm{c}}$ 
contain in themselves all physical processes from the excitation of a photon to the measurement in the detector. On the other hand, we assume that the radiation efficiencies are independent of the current (or equivalently, vertical tip position). We have simultaneously fitted the above equations to the uncharged and charged lines, leading to excellent agreement (cf. solid lines in Figure 4a).

To obtain the efficiency of charge to photon conversion, the photon rates were normalized by the tunnelling current and were plotted as a function of relative $z$ position of the tip (see Figure $4 \mathrm{~b}$ ) estimated from the Simmons rule. ${ }^{30}$ If the radiation efficiencies of charged and uncharged species were equal, the intensity of the charged species $X_{x}^{+}$(black dots in Figure $4 b$ ) should increase $\left(\Delta I_{\mathrm{c}}\right)$ by the same amount as the intensity of the uncharged species $Q_{x}$ (red dots in Figure $4 \mathrm{~b}$ ) decreases $\left(\Delta I_{\mathrm{u}}\right)$. In contrast, the increase of the intensity of $X_{x}^{+}$clearly exceeds the decrease of $Q_{x}$. The fit for the two spectral lines gives a ratio of $\eta_{\mathrm{c}} / \eta_{\mathrm{u}}=18.8$. In other words, we deduce the change in normalized intensities $\left(\Delta I_{\mathcal{c}}, \Delta I_{\mathrm{u}}\right)$ when we vary the tunneling parameters and thus change the populations $\Delta p_{\mathrm{u}}$ and $\Delta p_{c}$. As the molecule is either charged or uncharged, we know that $\Delta p_{\mathrm{u}}=-\Delta p_{\mathrm{c}}$ and that any difference in the change in intensity is related to $\eta_{\mathrm{c}}$ and $\eta_{\mathrm{u}}: \frac{\Delta I_{\mathrm{c}}}{\Delta I_{\mathrm{u}}}=-\frac{\eta_{\mathrm{c}}}{\eta_{\mathrm{u}}}$ and we detect $\approx 19$ times more photons per tunnelling electron from the charged molecule than from the uncharged molecule. For clarity, the population of the charged and uncharged state is also indicated in Figure 4b. While in this phenomenological description multiple aspects enter to explain the higher efficiency, a leading contribution can be assigned to the much higher efficiency to out couple a photon when it is vertically polarized relative to the substrate as compared to a horizontally polarized dipole (see SI Note 3). Even though the additional vertical dipole moment is rather modest, the out coupling efficiency is higher by 2 orders of magnitude, which permits us to extract more light into the far field.

In conclusion, we have demonstrated that atomic displace ments in charged molecules can largely boost the coupling to the far field. This effect offers valuable insights into the further design of molecules to enhance their emission in such junctions. Basically, we require them to offer a large vertical dipole moment in the involved states and in the presence of a tip. These findings give a clear guidance for future explorations in the field of computational material design to identify suitable molecules.

\section{- AUTHOR INFORMATION}

\section{Corresponding Author}

Vibhuti Rai - Institute for Quantum Materials and

Technologies, Karlsruhe Institute of Technology (KIT), D

76344 Eggenstein Leopoldshafen, Germany;

; Email: vibhuti.rai@kit.edu

\section{Authors}

Lukas Gerhard - Institute for Quantum Materials and Technologies, Karlsruhe Institute of Technology (KIT), D 76344 Eggenstein Leopoldshafen, Germany;

Qing Sun - Institute for Quantum Materials and Technologies, Karlsruhe Institute of Technology (KIT), D 76344 Eggenstein Leopoldshafen, Germany

Christof Holzer - Institute of Theoretical Solid State Physics, Karlsruhe Institute of Technology (KIT), D 76131 Karlsruhe, Germany;

Taavi Repän - Institute of Nanotechnology, Karlsruhe Institute of Technology (KIT), D 76344 Eggenstein Leopoldshafen, Germany

Marjan Krstic - Institute of Theoretical Solid State Physics, Karlsruhe Institute of Technology (KIT), D 76131 Karlsruhe, Germany

Liang Yang - Institute of Nanotechnology and Institute of Applied Physics, Karlsruhe Institute of Technology (KIT), D 76344 Eggenstein Leopoldshafen, Germany

Martin Wegener - Institute of Nanotechnology and Institute of Applied Physics, Karlsruhe Institute of Technology (KIT), D 76344 Eggenstein Leopoldshafen, Germany

Carsten Rockstuhl - Institute of Theoretical Solid State Physics and Institute of Nanotechnology, Karlsruhe Institute of Technology (KIT), D 76131 Karlsruhe, Germany

Wulf Wulfhekel - Institute for Quantum Materials and Technologies, Karlsruhe Institute of Technology (KIT), D 76344 Eggenstein Leopoldshafen, Germany

\section{Notes}

The authors declare no competing financial interest.

\section{ACKNOWLEDGMENTS}

This work was supported by the Helmholtz program Science and Technology of Nanosystems (STN), by the Deutsche Forschungsgemeinschaft (DFG, German Research Founda tion) under Germany's Excellence Strategy via the Excellence Cluster 3D Matter Made to Order (EXC 2082/1 390761711), by the Carl Zeiss Foundation through the "Carl Zeiss Focus@ HEiKA", and by the Volkswagen Foundation. V.R. gratefully acknowledges funding by the Deutsche Akademische Aus tauschdienst (DAAD). The authors are grateful to the company JCMwave for their free provision of the FEM Maxwell solver JCMsuite with which the simulations have been performed.

\section{REFERENCES}

(1) Coombs, J.; Gimzewski, J.; Reihl, B.; Sass, J.; Schlittler, R. Photon emission experiments with the scanning tunnelling micro scope. J. Microsc. 1988, 152, 325-336.

(2) Qiu, X.; Nazin, G.; Ho, W. Vibrationally resolved fluorescence excited with submolecular precision. Science 2003, 299, 542-546. 
(3) Chen, G.; Luo, Y.; Gao, H.; Jiang, J.; Yu, Y.; Zhang, L.; Zhang, Y.; Li, X.; Zhang, Z.; Dong, Z. Spin Triplet Mediated Up Conversion and Crossover Behavior in Single Molecule Electroluminescence. Phys. Rev. Lett. 2019, 122, 177401.

(4) Doppagne, B.; Chong, M. C.; Bulou, H.; Boeglin, A.; Scheurer, F.; Schull, G. Electrofluorochromism at the single molecule level. Science 2018, 361, 251-255.

(5) Miwa, K.; Najarian, A. M.; McCreery, R. L.; Galperin, M. Hubbard Nonequilibrium Green's Function Analysis of Photocurrent in Nitroazobenzene Molecular Junction. J. Phys. Chem. Lett. 2019, 10, $1550-1557$.

(6) White, A. J.; Tretiak, S.; Galperin, M. Raman scattering in molecular junctions: a pseudoparticle formulation. Nano Lett. 2014 14, 699-703.

(7) Miwa, K.; Imada, H.; Imai Imada, M.; Kimura, K.; Galperin, M.; Kim, Y. Many body state description of single molecule electro luminescence driven by a scanning tunneling microscope. Nano Lett. 2019, 19, 2803-2811.

(8) Dong, Z.; Zhang, X.; Gao, H.; Luo, Y.; Zhang, C.; Chen, L.; Zhang, R.; Tao, X.; Zhang, Y.; Yang, J.; et al. Generation of molecular hot electroluminescence by resonant nanocavity plasmons. Nat Photonics 2010, 4, 50.

(9) Zhang, Y.; Meng, Q. S.; Zhang, L.; Luo, Y.; Yu, Y. J.; Yang, B.; Zhang, Y.; Esteban, R.; Aizpurua, J.; Luo, Y.; Yang, J. L.; Dong, Z. C.; Hou, J G; et al. Sub nanometre control of the coherent interaction between a single molecule and a plasmonic nanocavity. Nat. Commun. $2017,8,15225$.

(10) Imada, H.; Miwa, K.; Imai Imada, M.; Kawahara, S.; Kimura, K.; Kim, Y. Single molecule investigation of energy dynamics in a coupled plasmon exciton system. Phys. Rev. Lett. 2017, 119, 013901.

(11) Zhang, Y.; Luo, Y.; Zhang, Y.; Yu, Y. J.; Kuang, Y. M.; Zhang, L.; Meng, Q. S.; Luo, Y.; Yang, J. L.; Dong, Z. C.; et al. Visualizing coherent intermolecular dipole-dipole coupling in real space. Nature 2016, 531, 623.

(12) Repp, J.; Meyer, G.; Stojković, S. M.; Gourdon, A.; Joachim, C. Molecules on insulating films: scanning tunneling microscopy imaging of individual molecular orbitals. Phys. Rev. Lett. 2005, 94, 026803.

(13) Edelmann, K.; Gerhard, L.; Winkler, M.; Wilmes, L.; Rai, V.; Schumann, M.; Kern, C.; Meyer, M.; Wegener, M.; Wulfhekel, W. Light collection from a low temperature scanning tunneling micro scope using integrated mirror tips fabricated by direct laser writing. Rev. Sci. Instrum. 2018, 89, 123107.

(14) Kuhnke, K.; Grosse, C.; Merino, P.; Kern, K. Atomic scale imaging and spectroscopy of electroluminescence at molecular interfaces. Chem. Rev. 2017, 117, 5174-5222.

(15) Imada, H.; Miwa, K.; Imai Imada, M.; Kawahara, S.; Kimura, K.; Kim, Y. Real space investigation of energy transfer in heterogeneous molecular dimers. Nature 2016, 538, 364.

(16) Bondybey, V.; English, J. Spectra of the h2 phthalocyanine in low temperature matrixes. J. Am. Chem. Soc. 1979, 101, 3446-3450.

(17) Murray, C.; Dozova, N.; McCaffrey, J. G.; Shafizadeh, N.; Chin W.; Broquier, M.; Crépin, C. Visible luminescence spectroscopy of free base and zinc phthalocyanines isolated in cryogenic matrices. Phys. Chem. Chem. Phys. 2011, 13, 17543-17554.

(18) Fitch, P. S.; Haynam, C. A.; Levy, D. H. The fluorescence excitation spectrum of free base phthalocyanine cooled in a supersonic free jet. J. Chem. Phys. 1980, 73, 1064-1072.

(19) Fukuda, R.; Ehara, M.; Nakatsuji, H. Excited states and electronic spectra of extended tetraazaporphyrins. J. Chem. Phys. 2010, $133,144316$.

(20) Adamo, C.; Barone, V. Toward reliable density functional methods without adjustable parameters: The PBE0 model. J. Chem. Phys. 1999, 110, 6158-6170.

(21) Weigend, F.; Ahlrichs, R. Balanced basis sets of split valence, triple zeta valence and quadruple zeta valence quality for $\mathrm{H}$ to $\mathrm{Rn}$ : Design and assessment of accuracy. Phys. Chem. Chem. Phys. 2005, 7, 3297-3305

(22) Balasubramani, S. G.; Chen, G. P.; Coriani, S.; Diedenhofen, M.; Frank, M. S.; Franzke, Y. J.; Furche, F.; Grotjahn, R.; Harding, M.
E.; Hättig, C.; et al. TURBOMOLE: Modular program suite for ab initio quantum chemical and condensed matter simulations. J. Chem. Phys. 2020, 152, 184107.

(23) Baeten, Y.; Fron, E.; Ruzié, C.; Geerts, Y. H.; Van Der Auweraer, M. Investigation of the Qx-Qy Equilibrium in a Metal Free Phthalocyanine. ChemPhysChem 2015, 16, 3992-3996.

(24) Doppagne, B.; Neuman, T.; Soria Martinez, R.; López, L. E. P.; Bulou, H.; Romeo, M.; Berciaud, S.; Scheurer, F.; Aizpurua, J.; Schull, G. Single molecule tautomerization tracking through space and time resolved fluorescence spectroscopy. Nat. Nanotechnol. 2020, 15, 207211

(25) Chen, C.; Chu, P.; Bobisch, C.; Mills, D.; Ho, W. Viewing the interior of a single molecule: vibronically resolved photon imaging at submolecular resolution. Phys. Rev. Lett. 2010, 105, 217402.

(26) Lee, J.; Perdue, S. M.; Rodriguez Perez, A.; Apkarian, V. A. Vibronic motion with joint angstrom-femtosecond resolution observed through Fano progressions recorded within one molecule. ACS Nano 2014, 8, 54-63.

(27) Doppagne, B.; Chong, M. C.; Lorchat, E.; Berciaud, S.; Romeo, M.; Bulou, H.; Boeglin, A.; Scheurer, F.; Schull, G. Vibronic spectroscopy with submolecular resolution from STM induced electroluminescence. Phys. Rev. Lett. 2017, 118, 127401.

(28) Neuman, T.; Esteban, R.; Casanova, D.; García Vidal, F. J.; Aizpurua, J. Coupling of molecular emitters and plasmonic cavities beyond the point dipole approximation. Nano Lett. 2018, 18, 23582364.

(29) Kügel, J.; Sixta, A.; Böhme, M.; Krönlein, A.; Bode, M. Breaking Degeneracy of Tautomerization Metastability from Days to Seconds. ACS Nano 2016, 10, 11058-11065.

(30) Simmons, J. G. Generalized formula for the electric tunnel effect between similar electrodes separated by a thin insulating film. J. Appl. Phys. 1963, 34, 1793-1803. 
Karlsruher Institut für Technologie

\section{Repository KITopen}

Dies ist ein Postprint/begutachtetes Manuskript.

Empfohlene Zitierung:

Rai, V.; Gerhard, L.; Sun, Q.; Holzer, C.; Repän, T.; Krstić, M.; Yang, L.; Wegener, M.; Rockstuhl, C.; Wulfhekel, W.

Boosting Light Emission from Single Hydrogen Phthalocyanine Molecules by Charging. 2020. Nano letters doi: $10.5445 / / R / 1000125692$

Zitierung der Originalveröffentlichung:

Rai, V.; Gerhard, L.; Sun, Q.; Holzer, C.; Repän, T.; Krstić, M.; Yang, L.; Wegener, M.; Rockstuhl, C.; Wulfhekel, W. Boosting Light Emission from Single Hydrogen Phthalocyanine Molecules by Charging. 2020. Nano letters, 20 (10), 7600-7605. doi:10.1021/acs.nanolett.0c03121 